\title{
Design and Fabrication of Photo-Responsive Hydrogel for the Application of Functional Contact Lens
}

OPEN ACCESS

Edited by:

Tiantian Kong,

Shenzhen University, China

Reviewed by:

Goutam Thakur

Manipal Institute of Technology, India

Xiao Gong,

Wuhan University of Technology,

China

*Correspondence:

Xiaohong $\mathrm{Hu}$

huxiaohong07@163.com

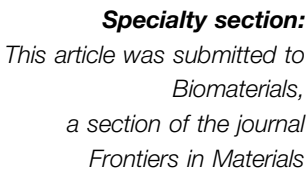

Received: 14 March 2021

Accepted: 22 June 2021

Published: 12 August 2021

Citation:

Lin X, Zhang H, Qin Y and Hu X (2021)

Design and Fabrication of Photo-

Responsive Hydrogel for the

Application of Functional

Contact Lens.

Front. Mater. 8:680359.

doi: $10.3389 /$ fmats.2021.680359

\author{
Xiaoxia Lin, Haitao Zhang, Yuqing Qin and Xiaohong $\mathrm{Hu}^{*}$
}

School of Material Engineering, Jinling Institute of Technology, Nanjing, China

Traditional hydrogel has usually had restricted application in the biomedical field due to lack of a control unit. Herein, Azobenzene (AZO), as a typical kind of photo-response molecule, was introduced into hydrogel by polymerization to provide a control unit for hydrogel. Simultaneously, the immobilization of AZO end group would solve the problems of uncontrollable recovery and light fatigue for AZO small molecules according to previous research. In the research, two kinds of crosslinkable AZO molecules, namely AZO crosslinker and AZO monomer, were synthesized as functional monomers or crosslinkers. Further, AZO copolymerized hydrogel and AZO crosslinked hydrogel were obtained. In order to estimate fundamental properties for contact lenses, gelation time and swelling ratio of hydrogels as a function of $\mathrm{AZO}$ concentration as well as crosslinking type were investigated. Moreover, synthesized hydrogels exhibited typical porous morphology, but their size and homogeneity were dependent on the type of hydrogel. In order to evaluate photo-responsive performance, detailed photo induced isomerization of both AZO copolymerized hydrogel and crosslinked hydrogel were tracked by UV spectra. The results confirmed the reversible, stable, and controllable photo responsive process. In vitro evaluation was used to investigate drug release behavior using orfloxacin and puerarin as model drugs. It was found that AZO addition as well as hydrogel type would influence puerarin release, but would have little effect on the orfloxacin release behavior in hydrogels. Furthermore, the isomer type in the hydrogel would have some effects on drug release behavior including orfloxacin and puerarin.

Keywords: hydrogel, azobenzene, hydrogel contact lens, drug delivery, isomerization

\section{INTRODUCTION}

Since the emergence of poly(hydroxyethyl methylacrylate) (pHEMA) hydrogel as soft contact lenses in the 1960s, the swollen soft materials became favorites in the materials field on account of having a similar microenvironment to the biological system (Hao et al., 2011; Hu and Gong, 2016; Hoteling et al., 2018; Samsom et al., 2018; Lorente-Velazquez et al., 2019; Wei et al., 2019). Traditional hydrogel contact lenses were synthesized by some hydrophilic monomers (containing such as hydroxy, carboxyl groups, different from hydrophobic groups like alkyl groups) like HEMA, vinyl pyrrolidone (NVP), acrylamide (AA) with excellent transparency, controllable water content, and certain non-deformability (Hoteling et al., 2018; Samsom et al., 2018; Lorente-Velazquez et al., 2019; Tong et al., 2019; Wei et al., 2019; Guan et al., 2020; Wei et al., 2020). However, these early 
synthesized hydrogels had some original drawbacks due to their structure and properties. Typically, an aqueous microenvironment also benefits bacterial reproduction, which would induce eye infection (Hoteling et al., 2018; Samsom et al., 2018; Lorente-Velazquez et al., 2019; Wei et al., 2019). Recently, with the development of regeneration medicine, traditional hydrogels were explored for new applications as drug carriers/ hydrogel scaffolds (Hao et al., 2011; Hu and Gong, 2016; Hoteling et al., 2018; Samsom et al., 2018; Lorente-Velazquez et al., 2019; Wei et al., 2019). While these new applications put forward new requests for tradition hydrogels, among these requests, the control unit seems especially relevant for its application.

Generally, the control unit is composed of a stimuli-response structure or material. Until now, the $\mathrm{pH}$-responsive unit, photoresponsive unit, electric-responsive unit, magnetic responsive unit, and chemical responsive unit have been investigated as control units (Dong and Chen, 2011; Guragain et al., 2015; Kim et al., 2015; Liu et al., 2015; Lorenzo et al., 2015; Wang et al., 2015; Bian et al., 2016a; Cao and Wang, 2016; Dong et al., 2017; Xiao et al., 2019). Azobenzene (AZO) has photoisomerizing characteristic, which provides possibilities as a photo switch (Li et al., 2014; Pang et al., 2014). However, uncontrollability and instability of photoisomerization for azobenzene and its small derivatives restricted applications of the chemicals as photo switches. Therefore, many measures have been made to improve the controllability and stability of photoisomerization including fixing one end of molecule to a solid surface, grafting onto a polymer chain, copolymerization with other monomer, and introduction of the long chain to one or both ends of AZO (Deka et al., 2015; Bian et al., 2016a; Bian et al., 2016b; Pang et al., 2018; Gao et al., 2019; Pang et al., 2020a; Pang et al., 2020b; Chen et al., 2020). In these contributions, our research mainly focused on the introduction of AZO to polymer by grafting, copolymerization, and $\mathrm{AZO}$ initiated ring opening polymerization (ROP) (Chen et al., 2020). Once AZO molecules were combined with polymer, photoisomerization of AZO domain could be adjusted by either the external environment of macromolecules including solvent, temperature, light density, light source, or internal structure including properties of copolymer monomer and chain length of polymer (Pang et al., 2018; Gao et al., 2019; Pang et al., 2020b). Furthermore, AZO based macromolecule could be endowed with biocompatibility according to the properties of the main chain (Pang et al., 2018). When it comes to application of photo switches, material is an indispensable carrier. Therefore, the AZO based molecules have been fabricated into nanoparticles, films, hydrogels, etc.

It was inferred from existing research that time-dependent restricted motion of polymers provides better controllability of AZO domain isomerization transformation and stability of AZO isomers, especially for cis-isomers. Besides time, spatial conditions were also inferred to be optimal elements for improving controllability and stability of photoisomerization. Therefore, the AZO unit was introduced to the traditional hydrogel network by copolymerization in the research. In one aspect, the AZO unit was restricted in a mesh of hydrogel network, which would improve the controllability and stability of AZO photoisomerization. In other aspects, AZO photoisomerization would bring the deformation of mesh in the hydrogel network, which would endow photo-response properties to tradition hydrogel. In order to realize the idea, AZO crosslinked hydrogel and AZO copolymeried hydrogel were synthesized and investigated elaborately in the research. Finally, the function of drug delivery was evaluated to explore new application of tradition hydrogel in the work.

Experiment

\section{MATERIALS}

Acryloyl chloride, p-aminoazobenzene (AZO), and azodianiline (DAZO) was purchased from Aladdin. Hydroxyethyl methacrylate (HEMA), N-Vinyl pyrrolidone (NVP) and puerarin were obtained from Shanghai Jingchun Industries Co. Ltd., China and distilled under vacuum before use. Dichloromethane (DCM), diethyl ether, tetrahydrofuran (THF), dioxane, triethylamine (TEA) and dimethylsulfone (DMSO), ammonium persulfate (APS), and $\mathrm{N}, \mathrm{N}, \mathrm{N}^{\prime}, \mathrm{N}^{\prime}$ tetramethylethylenediamine (TEMED) were obtained from Shanghai Chemical Industries Co. Ltd., (China). Hyaluronic acid and fluorescein isothiocyanate were obtained from Sigma. Norfloxacin and puerarin were purchased from Jinling pharmac Industries Co. Ltd., China. All other reagents and solvents were of analytical grade and used as received.

\section{Synthesis of AZO Monomer and AZO Crosslinker}

AZO monomer and AZO crosslinker were synthesized by acylchlorination (Gao et al., 2019). AZO monomer was synthesized according to the previous method. Briefly, $10 \mathrm{mM}$ AZO was reacted with $11 \mathrm{mmol}$ acryloyl chloride in $20 \mathrm{ml}$ anhydrous DCM at stirred state at room temperature for 4-5 h, using TEA as an alkali absorber. After reaction, AZO monomer was obtained by the water washing purification procedure and reduced pressure distillation. AZO crosslinker was synthesized using a similar method. Briefly, $10 \mathrm{mM}$ DAZO was reacted with $33 \mathrm{mmol}$ acryloyl chloride in $20 \mathrm{ml}$ DMF at stirred state at room temperature for $20 \mathrm{~h}$, using TEA as an alkali absorber. Differently, the obtained product was collected by precipitation with water $(\mathrm{pH} 4)$ and washed with saturated $\mathrm{NaHCO}_{3}$ solution and water. The final product was obtained by freeze-drying and characterized by ${ }_{1} \mathrm{H}$ nuclear magnetic resonance $\left({ }_{1} \mathrm{H}\right.$ NMR, Bruker, AV300).

\section{Synthesis and Characterization of Hydrogels}

AZO copolymerized hydrogel and AZO crosslinked hydrogel were fabricated by the radical polymerization. Briefly, AZO monomer/AZO crosslinker with various ratios dissolved into $5 \mathrm{ml}$ HEMA and $1.162 \mathrm{ml} \mathrm{NVP}$ by stirring, into which certain amounts of APS and TEMED with equal molar ratio were respectively added with final initiator concentration at $0.5 \mathrm{~mol}$ 
$\%$. The above mixture was injected into a circle model, and then polymerized at $60^{\circ} \mathrm{C}$ in oven. Gelation time was obtained by judgment of its fluidity. In order to ensure full crosslinking, $1 \mathrm{~h}$ polymerization time was used in the hydrogel product.

Next, the fundamental properties of the hydrogels like swelling ratio and hydrogel morphology as contact lenses were characterized. Hydrogels were dried and weighed $\left(W_{0}\right)$. The dried hydrogels had been swollen to equilibrium in water and weighed $\left(W_{1}\right)$. The equilibrium swelling ratio of the hydrogels was defined as $W_{0} / W_{1} \times 100 \%$. The morphology of the hydrogels was characterized by scanning electron microscope (SEM, S$8100)$.

\section{Responsive and Recovery Performance of Hydrogels}

Hydrogels were tracked by UV spectroscopy (Cary 50). UV lamp $(10 \mathrm{~W})$ was used as a photo source to induce trans-to-cis transition of AZO domain in hydrogels. After UV irradiation, white light was used to induce cis-to-trans recovery at different conditions. Real-time UV spectra as a function of irradiation time and recovery time were recorded to track the structural change of molecules. Repeated irradiation and recovery methods were used to investigate fatigue resistance of AZO domain in hydrogels. Moreover, factors of temperature and light density were applied to clarified photo-isomerization for AZO domain in hydrogel.

\section{In vitro Drug Delivery Evaluation}

Orfloxacin and puerarin were used as model drugs to be loaded into hydrogel by copolymerization in situ. Briefly, orfloxacin and puerarin was dissolved in monomers of HEMA and NVP with concentration of $3 \mathrm{mg} / \mathrm{ml}$. Then hydrogel with drugs was formed using the above-mentioned method.

The above-prepared hydrogels were submerged in $4 \mathrm{ml}$ PBS in a $5 \mathrm{ml}$ centrifugal tube, which was placed under shake cultivation at specific temperature. At regular intervals, $2 \mathrm{ml}$ of released solution were moved for detection. Simultaneously, $2 \mathrm{ml}$ of fresh PBS were supplemented into the released system. At given times, the absorbance of removed drug solution at $280 \mathrm{~nm}$ for orfloxacin and at $355 \mathrm{~nm}$ for puerarin was recorded by UV spectroscopy. The drug concentration was obtained by dilution ratio and a calibration curve, which was constructed from known concentrations of drug solutions. The cumulative released amount was then calculated according to drug concentration and volume. UV light was used to excite isomerization of AZO crosslinked hydrogel in the process of drug release. After excitation, drug release was performed in a dark environment at $20^{\circ} \mathrm{C}$.

\section{Statistical Analysis}

Data were analyzed using the t-test for differences. Results are reported as means \pm standard deviation. The significant level was set at $p<0.05$.

\section{RESULTS AND DISCUSSION}

\section{Fabrication and Characterization of Photo-Responsive Hydrogel}

The AZO crosslinker was synthesized by acylchlorination, which was characterized by ${ }_{1} \mathrm{H}$ NMR spectrum in Figure 1. In detail, chemical shifts from 5.8 to 6.5 ppm were attributed to hydrogens of vinyl groups at a, b, and c positions, chemical shifts from 7.1 to $7.9 \mathrm{ppm}$ are attributed to $\mathrm{Hs}$ of benzene ring, and chemical shifts at $10.5 \mathrm{ppm}$ are attributed to $\mathrm{H}$ of imine groups. Besides qualitative analysis, quantitative calculation was applied to confirm the precise chemical structure of the AZO crosslinker. The integration of peak from 5.8 to $6.5 \mathrm{ppm}$ was 3.0 , the integration of peak from 7.1 to $7.9 \mathrm{ppm}$ was 4.0 , the integration of peak at $10.5 \mathrm{ppm}$ was 1.0 . These ratios were consistent with $\mathrm{H}$ numbers of groups, which are listed as $6 \mathrm{Hs}$ for vinyl, $8 \mathrm{Hs}$ for benzene ring, $2 \mathrm{Hs}$ for imine. These results indicated that AZO crosslinker with two vinyls was successfully synthesized without any impurity.

In order to endow hydrogel photo-response property, two kinds of hydrogel were synthesized by radical polymerization, namely AZO copolymerized hydrogel and AZO crosslinked hydrogel as shown in Figures 2A,B. Formed hydrogels exhibited a yellow transparent state (Figures 2A,B). Since traditional hydrogel was colorless, yellow color could demonstrate the successful synthesis of AZO copolymerized hydrogel and AZO crosslinked hydrogel from a side. In polymerization, AZO domain introduction would influence the gelation time and swelling ratio (Figures 2C,D). For AZO copolymerized hydrogel, the gelation time was prolonged largely by the increased AZO monomer (Figure 2C). Even the hydrogel could not be gelled when the AZO monomer concentration exceeded 4\%. For AZO crosslinked hydrogel, the gelation time was also prolonged by the increased AZO crosslinker (Figure 2C). Similarly, the hydrogel could hardly be gelled when AZO crosslinker concentration exceeded 4\%. Since gelation of pHEMA kind hydrogel depended on the growth of branch and mutual crosslinking between branches, the steric hindrance of the AZO structure would hinder branch formation in polymerization. Thus, the gelation time would increase along with the AZO domain increasing. Furthermore, gelation time of AZO crosslinked hydrogel was shorter than that of AZO copolymerized hydrogel with significant difference on account of the larger double carbon bond density (Figure 2C). Differently, the swelling ratio of both AZO crosslinked hydrogel and AZO copolymerized hydrogel decreased with the increase of AZO domain in hydrogel (Figure 2D). When the AZO domain reached $4 \%$, the swelling ratio of AZO crosslinked hydrogel was significantly smaller than that of AZO copolymerized hydrogel (Figure 2D). Since the AZO domain was not a hydration unit, the hydrogel with the AZO domain decreased sight with the increase of AZO domain. Moreover, crosslinker density was another factor to influence the swelling ratio of hydrogels. Therefore, the AZO crosslinked hydrogel possessed a smaller swelling ratio due to larger crosslinker density. 


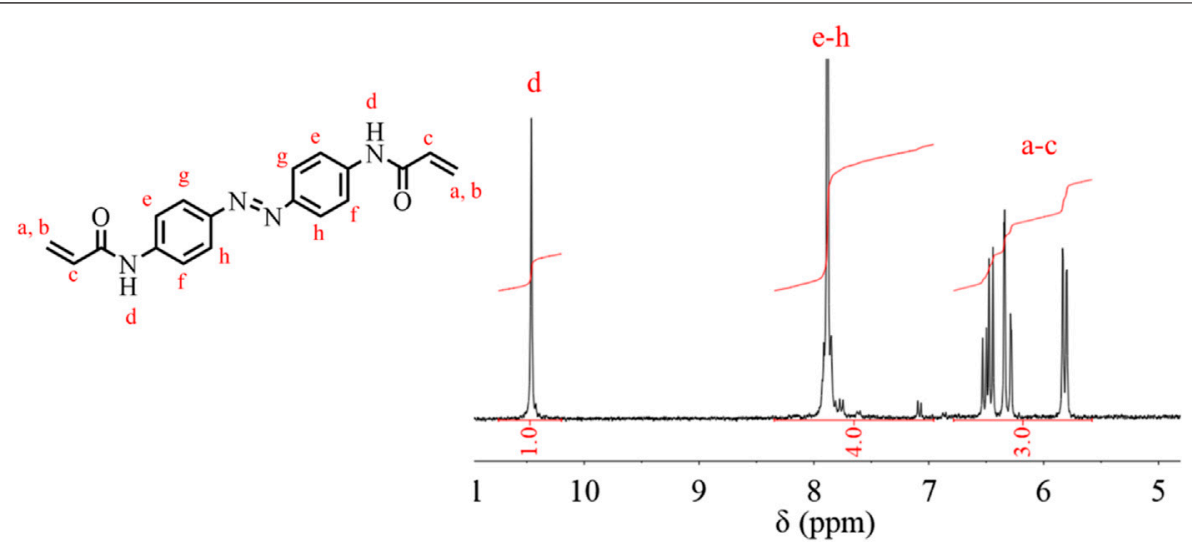

FIGURE 1 | ${ }_{1} \mathrm{H}$ NMR spectrum of AZO crosslinker.

A<smiles>[CH]CC(=C)C(=O)OCCO</smiles><smiles>C=CN1CCCC1=O</smiles>

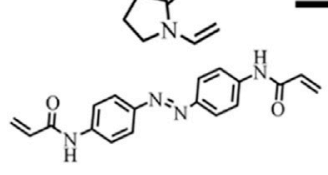

C

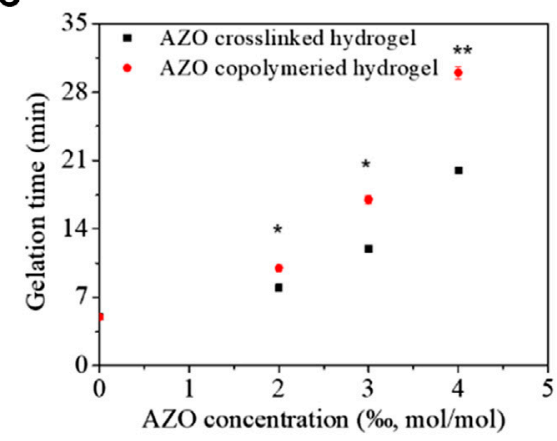

B

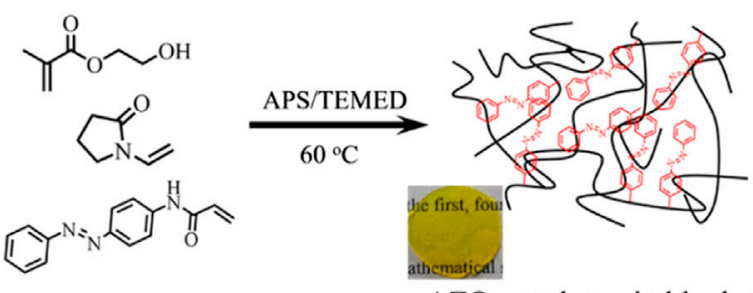

AZO copolymeried hydrogel

D

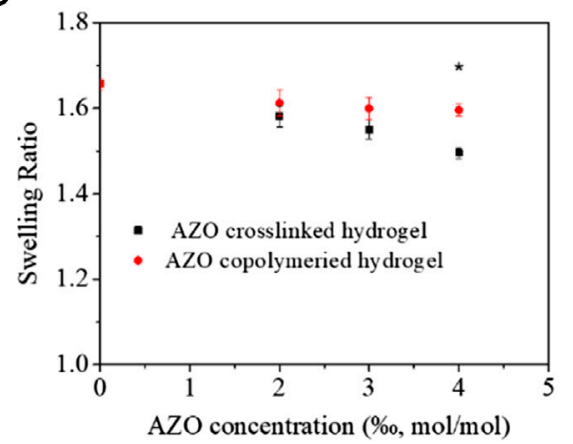

FIGURE 2 | Schematic formation of AZO crosslinked hydrogel (A) and AZO copolymerizated hydrogel (B). (C) gelation time of hydrogel as a function of AZO concentration. (D) swelling ratio of hydrogel as a function of AZO concentration. ${ }^{*} p<0.05,{ }^{* *} p<0.01$.
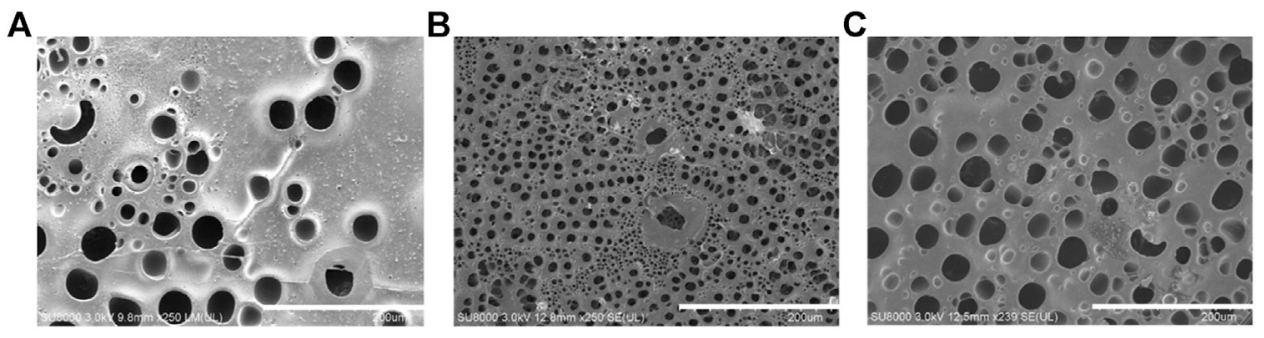

FIGURE 3 | SEM images of traditional hydrogel (A), AZO crosslinked hydrogel (B) and AZO copolymerizated hydrogel (C). The scale bar is $200 \mu \mathrm{m}$. 

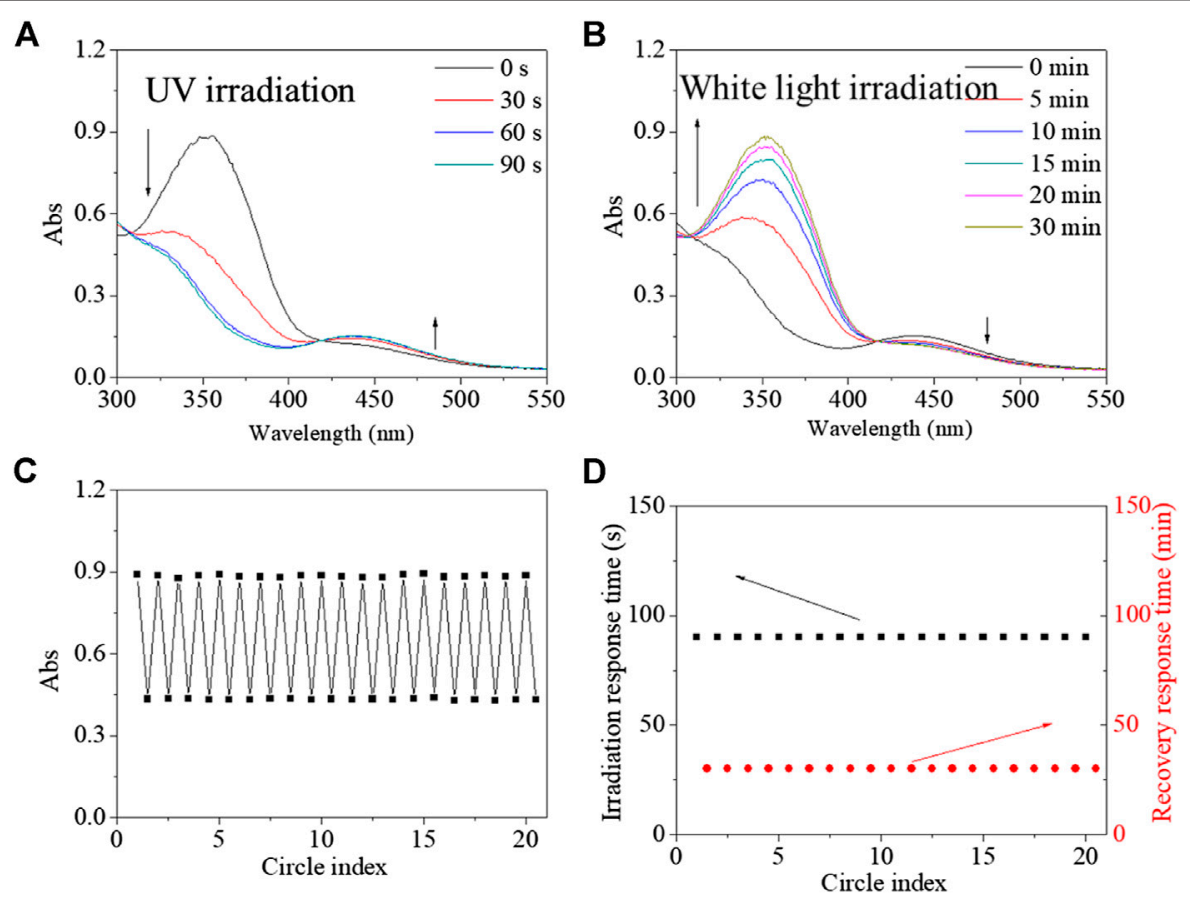

FIGURE 4 I UV spectra of AZO copolymerizated hydrogel as a function of irradiation time (A) and recovery time (B). (C) Absorbance at $350 \mathrm{~nm} / 330 \mathrm{~nm}$ of $\mathrm{AZO}$ copolymerizated hydrogel as a function of circle index. (D) Irradiation response time by UV and recovery response time by white light as a function of circle index. Light density of white light was set at 1,000 Lux and room temperature.

Moreover, hydrogel morphology was characterized by SEM images to clarify hydrogel inner structure, which was shown in Figure 3. Hydrogels exhibited a typical porous structure, but porous size was different for different hydrogels. Namely, the traditional hydrogel possessed the largest pore size and an asymmetrical structure. The AZO crosslinked hydrogel exhibited the smallest pore size and a homogeneous structure. The AZO copolymerized hydrogel had a medium pore size and a relative homogeneous structure. The hydrophilic and soft chain nature of traditional hydrogel would collapse during freeze-drying, which resulted in asymmetrical structure. However, AZO domain introduction would increase the rigidity of the polymer chain, which prevented the hydrogel from collapsing during freezedrying. For the AZO crosslinked hydrogel, larger crosslink density resulted in smaller mesh size in the hydrogel. These results were consistent with the above-mentioned discussion.

\section{Photo Responsive Performance of Hydrogel}

As a photo-responsive unit, the photo-response of hydrogel was evaluated by UV spectrum using UV light as a stimulus since the trans isomer and cis isomer of the AZO unit had different UV spectra from the verified theory and previous research (Pang et al., 2018; Gao et al., 2019; Pang et al., 2020a; Pang et al., 2020b).

UV spectra of AZO copolymerized hydrogel as a function of irradiation time and recovery time were shown in Figures 4A,B. Originally, a typical absorption peak at $350 \mathrm{~nm}$ belonging to $\pi-\pi^{*}$ transition and a small flat absorption peak at $430 \mathrm{~nm}$ belonging to $\mathrm{n}-\pi^{\star}$ transition exhibited the typical characteristic of trans isomer of AZO unit in Figure 4A, which also confirmed the successful synthesis of AZO copolymerized hydrogel from the other side. After UV irradiation, the maximum absorbance at $350 \mathrm{~nm}$ decreased greatly and shifted to $330 \mathrm{~nm}$, simultaneously the absorbance at $430 \mathrm{~nm}$ increased a little with irradiation time until $90 \mathrm{~s}$, which indicated that the trans isomer had been transferred to the cis isomer (Figure 4A). After removing light, the recovery process from cis isomer to trans isomer could be accelerated by white light irradiation according to previous research (Pang et al., 2018; Gao et al., 2019; Pang et al., 2020a; Pang et al., 2020b). In order to track the recovery process in a convenient and observable period, white light irradiation was used to induce the recovery process from cis to trans in Figure 4B. Upon white light irradiation, absorbances at $330 \mathrm{~nm}$ were gradually shifted to $350 \mathrm{~nm}$ and recovered to their respective origin value within $30 \mathrm{~min}$, absorbance at $430 \mathrm{~nm}$ was also recovered to its origin value. These phenomena confirmed the reversible recovery of the trans structure (Figure 4B). Since absorbance at $350 \mathrm{~nm} / 330 \mathrm{~nm}$ directly reflected the trans isomer content and response time reflected the efficiency of the light control unit, the two parameters were used to evaluate fatigue resistance of copolymer repeated UV/white light irradiation, which was important for the photo control unit in Figures 4C,D. From the recorded real-time absorbance at $350 \mathrm{~nm} /$ $330 \mathrm{~nm}$ of AZO copolymerized hydrogel in Figure 4C, maximum absorbance at $350 \mathrm{~nm}$, either initially or after recovery, was stabilized around 0.9 regardless of circle time, and minimum absorbance at $330 \mathrm{~nm}$ after UV irradiation was stabilized at $0.4-0.5$ regardless of circle time. From the tracked response time in Figure 4D, irradiation response time was stable 

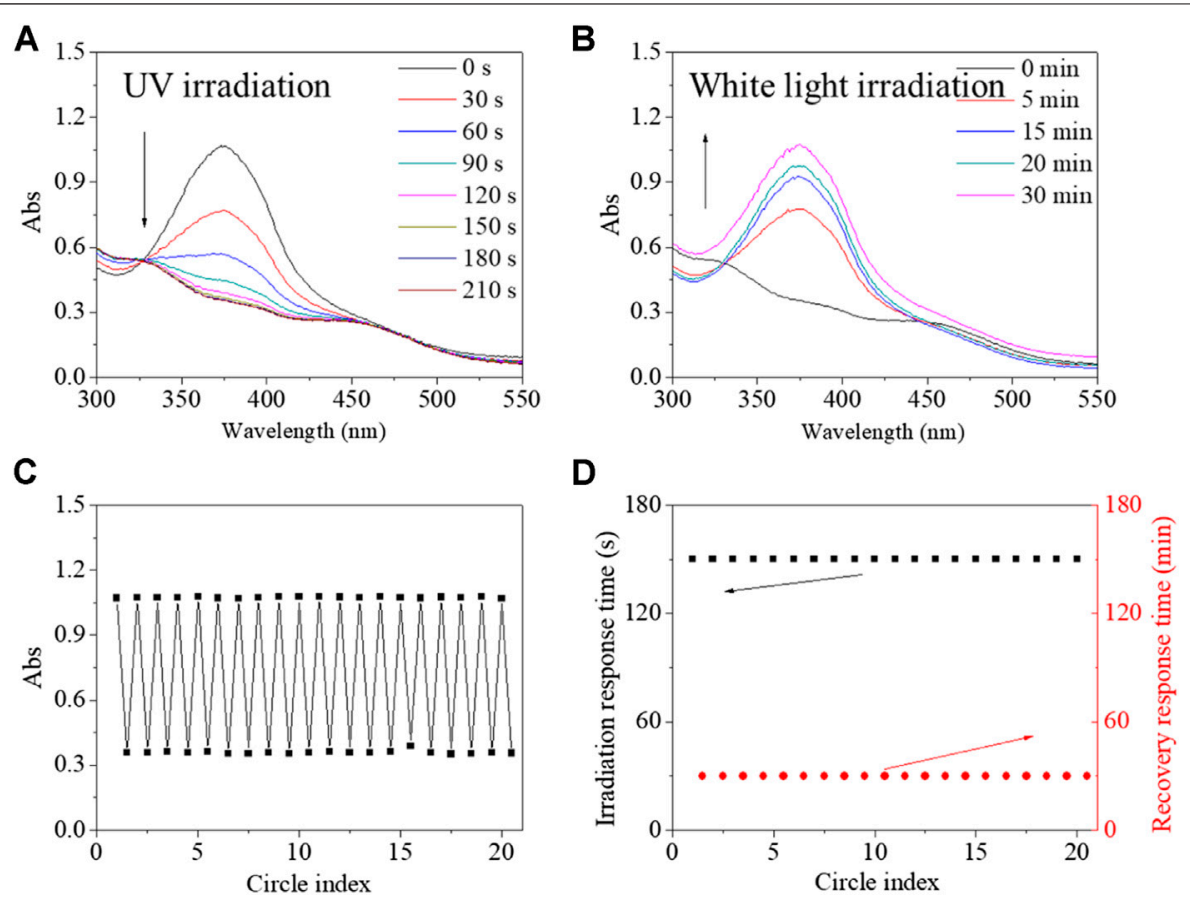

FIGURE 5 | UV spectra of AZO crosslinked hydrogel as a function of irradiation time (A) and recovery time (B). (C) Absorbance at 373 nm of AZO crosslinked hydrogel as a function of circle index. (D) Irradiation response time by UV and recovery response time by white light as a function of circle index. Light density of white light was set at 1,000 Lux and room temperature.

at $90 \mathrm{~s}$ and recovery response time was stable at $30 \mathrm{~min}$ regardless of circle time. These results confirmed that UV could induce reversible isomerization for the AZO copolymerized hydrogel, and further the transition could be realized stably by specific light excitation source without any sign of fatigue or photobleaching.

Besides the AZO copolymerized hydrogel, the reversible photo triggered isomerization of AZO crosslinked hydrogel was tracked by UV spectra in Figure 5. In its natural state, a typical absorption peak at $373 \mathrm{~nm}$ existed in the UV spectrum of AZO crosslinked hydrogel in Figure 5A, indicating the trans isomer of the AZO unit in hydrogel. After UV irradiation, the maximum absorbance decreased greatly until $150 \mathrm{~s}$, which indicated that the trans isomer had been transferred to the cis isomer completely (Figure 5A). Upon white light irradiation, absorbances at $373 \mathrm{~nm}$ recovered to their respective origin value within $30 \mathrm{~min}$, which confirmed the reversible recovery of the trans structure (Figure 5B). Moreover, maximum absorbance after white light irradiation was stabilized around 1.1 and minimum absorbance after UV irradiation was stabilized around 0.4 regardless of cycle number (Figure 5C). Simultaneously, irradiation response time was stable at $150 \mathrm{~s}$ and recovery response time was stable at $30 \mathrm{~min}$ regardless of circle time (Figure 5D). These results revealed that AZO crosslinked hydrogel possessed reversible and stable photo-responsive performance without photobleaching. Theoretically, the AZO domains existed in two ground states (trans and cis form) and one excited state. Transition between isomers must go through the excited state, although the excited state nearly could not be detected. Therefore, light excitation in fact provided energy between the ground state and excited state. Besides the energy factor, steric hindrance was also an important factor to influence isomerization transition from previous research (Pang et al., 2018; Gao et al., 2019; Pang et al., 2020a; Pang et al., 2020b). Although the transition energy of the AZO unit from trans to cis in the AZO crosslinked hydrogel is similar to that in the AZO copolymerized hydrogel, the AZO unit in the AZO crosslinked hydrogel with two fixed ends had a larger steric hindrance in the process of trans-to-cis transition than that of AZO copolymerized hydrogel with one fixed end. Therefore, in the same UV irradiated intensity, trans-to-cis transition of the AZO unit in the AZO crosslinked hydrogel needed a longer time (150s) to be completed than that of the AZO copolymerized hydrogel (30 s).

Since the recovery process is an essential factor to realize the controllable and stable photo response properties of hydrogel, it was investigated considering the influencing factors of light intensity and temperature in Figure 6. For the AZO copolymerized hydrogel, recovery time decreased greatly with the increase of light intensity, especially when light intensity was lower than 500 LUX (Figure 6A). Likewise, the recovery time of the AZO crosslinked hydrogel as a function of light intensity exhibited similar trends, except the trend became sharper than that of the AZO copolymerized hydrogel (Figure 6B). Additionally, the cis structure in both AZO copolymerized hydrogel and AZO crosslinked hydrogel could not be recovered in the dark during the observed 3 days at $20^{\circ} \mathrm{C}$, which was not listed in Figures 6A,B. Besides light intensity, effects of temperature were also presented in Figures 6C,D in consideration of two different light intensities (500 LUX and 

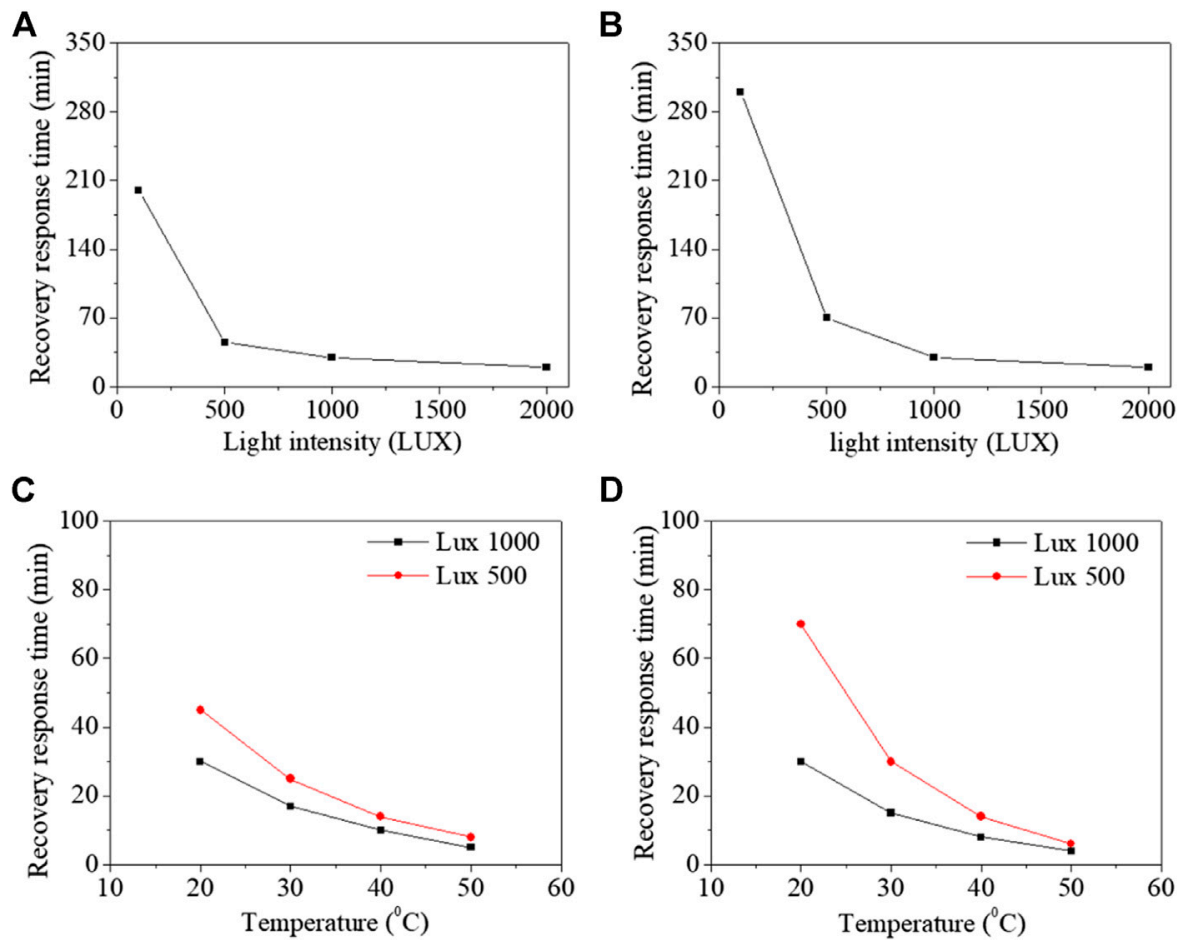

FIGURE 6 | The recovery response time as a function of light intensity (A, B) and temperature (C, D) for AZO copolymerized hydrogel (A, C) and AZO crosslinked hydrogel (B, D). Temperature was set at $20^{\circ} \mathrm{C}$ for (A, B); Light density of white light was set at 500 Lux and 1,000 Lux, respectively.
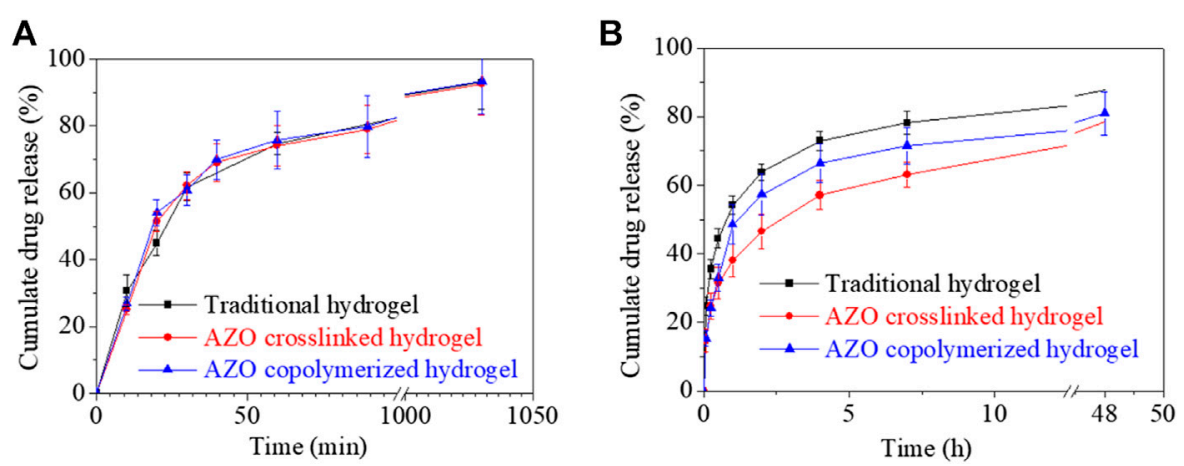

FIGURE 7 | The cumulate orfloxacin release (A) and cumulate puerarin release (B) of traditional hydrogel, AZO crosslinked hydrogel and AZO copolymeried hydrogel. The environment temperature was $37^{\circ} \mathrm{C}$

1,000 LUX). For the AZO copolymerized hydrogel, recovery time decreases gradually with the increase of temperature either under 500 LUX white light irradiation or 1,000 LUX white light irradiation (Figure 6C). Likewise, recovery time of AZO crosslinked hydrogel as a function of temperature exhibited similar trends with a sharper trend (Figure 6D). From the above discussion, the cis-to-trans recovery process should overcome the barrier between the cis ground state and excited state. While heat/white light could provide needed energy from the cis structure ground state to excited state. Therefore, it was easy to understand that higher temperature and light intensity resulted in shorter recovery response time.

In summary, both exhibited controllable and stable photo responsive performance, which could be adjusted by environmental factors.

\section{In vitro Drug Delivery Evaluation}

Since one important application of hydrogel contact lenses was as an ophthalmic drug carrier, performances of hydrogels as a kind of drug carrier were evaluated in vitro. Model drugs were incorporated into hydrogels by in situ polymerization. The cumulative drug release in different hydrogels is shown in 

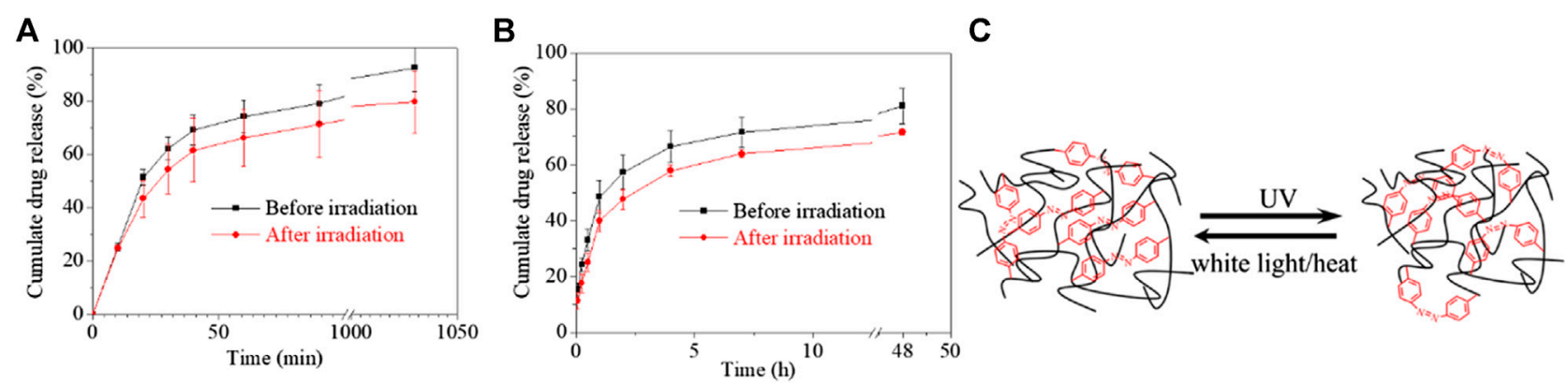

FIGURE 8 | The cumulate orfloxacin release (A) and cumulate puerarin release (B) of before irradiation and after irradiation of AZO crosslinked hydrogel. (C) Schematic illustratation of AZO crosslinked hydrogel response to UV/white light. The environment temperature was $20^{\circ} \mathrm{C}$

Figure 7. From Figure 7A, 70\% orfloxacin was rapidly released from any of hydrogels at initial $30 \mathrm{~min}$, and then another $20 \%$ orfloxacin was gradually released from any of hydrogels in the following $16-17 \mathrm{~h}$. Further, the difference either between traditional hydrogel and AZO functional hydrogel or between AZO copolymerized hydrogel and AZO crosslinked hydrogel was not significant. It seemed the slight addition of the AZO unit in the hydrogel network had little effect on orfloxacin release behavior. When it came to puerarin, the condition was different in Figure 7B. Initially, 30\% puerarin was rapidly released from any of hydrogels during the first $30 \mathrm{~min}$. Next, another $40 \%$ puerarin was relatively rapidly released from the traditional hydrogel, another $30 \%$ puerarin was relatively rapidly released from the AZO copolymerized hydrogel, and another $20 \%$ puerarin was relatively rapidly released from the AZO crosslinked hydrogel in the following $4-5 \mathrm{~h}$. Finally, an additional $10 \%$ puerarin was relatively slowly released from any of hydrogels. The delayed puerarin release in AZO containing hydrogel might be ascribed to the interaction between puerarin molecules and the AZO unit. The AZO crosslinked hydrogel possessed a more compact structure due to higher double carbon density, which resulted in a slower puerarin release rate.

Furthermore, the AZO crosslinked hydrogel was chosen as a carrier for drug release behavior investigation since isomerization of the AZO crosslinker in the hydrogel would cause deformation of the hydrogel network. Before the beginning of drug release, one group of hydrogels was irradiated by UV to realize trans-to-cis transition. After irradiation, drug release was performed in a dark environment at room temperature in order to maintain the cis structure of the AZO unit in hydrogel since the cis isomer could not be recovered to the trans isomer within 3 days from the above-mentioned discussion. The drug release behavior of two groups of AZO crosslinked hydrogels with or without irradiation were shown in Figures 8A,B. Without irradiation, the cumulate orfloxacin release behavior at room temperature in Figure 8A was similar to that at $37^{\circ} \mathrm{C}$ in Figure 7A. But after irradiation, the orfloxacin release speed became slower and the cumulative orfloxacin release amount became slightly lower without great significance in Figure 8A. For puerarin, it could gradually release from the hydrogel within $48 \mathrm{~h}$ after the first hour burst release at room temperature in its natural state (Figure 8B), which was also similar to that at $37^{\circ} \mathrm{C}$ in Figure $7 \mathbf{B}$. Likewise, the puerarin release speed became slower and cumulative orfloxacin release amount became obviously lower (Figure 8B). It was inferred from these results that trans-to-cis isomerization would make the crosslinked polymer network shrunken as Figure 8C showed, which resulted in a tighter mesh of the hydrogel network. Therefore, the cis-state of the AZO unit in the AZO crosslinked hydrogel delayed drug release.

\section{CONCLUSION}

Divinyl functional AZO, namely AZO crosslinker, was successfully synthesized without any impurities, just as the previously synthesized vinyl functional AZO (AZO monomer). Based on traditional hydrogel contact lenses, AZO functional hydrogels, namely AZO copolymerized hydrogel and AZO crosslinked hydrogel, were successfully copolymerized with an adjustable AZO unit (1-4\% W/V). Both the formed AZO copolymerized hydrogel and AZO crosslinked hydrogel exhibited a yellow transparent state. In polymerization, the gelation time was prolonged largely by the increased AZO unit for both hydrogels, but the effects of AZO were more significant for AZO crosslinked hydrogel than for AZO copolymerized hydrogel, especially when AZO concentration was higher than $2 \%$. The swelling ratio of both hydrogels decreased slightly with the increase of the AZO unit. Especially when the AZO domain reached 4\%, AZO crosslinked hydrogel possessed a significantly lower swelling ratio than the AZO copolymerized hydrogel. Synthesized hydrogels exhibited typical porous morphology, but their size and homogeneity were dependent on the type of hydrogel. UV could induce reversible isomerization of the AZO unit in both the AZO copolymerized hydrogel and crosslinked hydrogel, and the transition could further be realized stably by a specific light excitation source without any sign of fatigue or photobleaching. For the cis-to-trans process, high temperature and light intensity would accelerate transition speed. In summary, both exhibited controllable and stable photo responsive performance, which could be adjusted by environmental factors. In the in vitro evaluation, it was found that the slight addition of the AZO unit in the hydrogel network had little effect on the orfloxacin release behavior, but AZO unit would slow and delay puerarin release in hydrogel. Additionally, the more compact structure of the AZO crosslinked hydrogel could 
cause puerarin release more slowly. Furthermore, the cis state in the AZO crosslinked hydrogel would delay drug release either for orfloxacin and puerarin, which confirmed that the crosslinked polymer network would shrink in the cis state of AZO in the AZO crosslinked hydrogel.

\section{DATA AVAILABILITY STATEMENT}

The original contributions presented in the study are included in the article/supplementary material, further inquiries can be directed to the corresponding author.

\section{REFERENCES}

Bian, Q., Wang, W., Han, G., Chen, Y., Wang, S., and Wang, G. (2016). Photoswitched Cell Adhesion on Azobenzene-Containing Self-Assembled Films. ChemPhysChem 17, 2503-2508. doi:10.1002/cphc.201600362

Bian, Q., Wang, W., Wang, S., and Wang, G. (2016). Light-Triggered Specific Cancer Cell Release from Cyclodextrin/Azobenzene and Aptamer-Modified Substrate. ACS Appl. Mater. Inter. 8, 27360-27367. doi:10.1021/acsami.6b09734

Cao, Z.-Q., and Wang, G.-J. (2016). Multi-Stimuli-Responsive Polymer Materials: Particles, Films, and Bulk Gels. Chem. Rec. 16, 1398-1435. doi:10.1002/ tcr.201500281

Chen, P., Kong, J., Wang, X., Ma, W., Yang, X., Qin, Y., et al. (2020). Front. Chem. 8 (ID), 591. doi:10.3389/fchem.2020.00591

Deka, S. R., Yadav, S., Mahato, M., and Sharma, A. K. (2015). Azobenzene-aminoglycoside: Self-Assembled Smart Amphiphilic Nanostructures for Drug Delivery. Colloids Surf. B: Biointerfaces 135, 150-157. doi:10.1016/j.colsurfb.2015.07.026

Dong, C.-M., and Chen, Y. (2011). Stimuli-responsive Polypeptide-Based Reverse Micellar Hydrogel. J. Controlled Release. 152 (Suppl. 1), e13-e14. doi:10.1016/ j.jconrel.2011.08.092

Dong, M., Babalhavaeji, A., Collins, C. V., Jarrah, K., Sadovski, O., Dai, Q., et al. (2017). Near-Infrared Photoswitching of Azobenzenes under Physiological Conditions. J. Am. Chem. Soc. 139, 13483-13486. doi:10.1021/jacs.7b06471

Gao, Z. Y., Pang, J., Tan, H. P., Mao, X. C., Wang, H. M., and Hu, X. H. (2019). Front. Chem. 7 (ID), 86.

Guan, F., Song, Z., Xin, F., Wang, H., Yu, D., Li, G., et al. (2020). Preparation of Hydrophobic Transparent Paper via Using Polydimethylsiloxane as Transparent Agent. J. Bioresources Bioproducts 5, 37-43. doi:10.1016/j.jobab.2020.03.004

Guragain, S., Bastakoti, B. P., Malgras, V., Nakashima, K., and Yamauchi, Y. (2015). Multi-Stimuli-Responsive Polymeric Materials. Chem. Eur. J. 21, 13164-13174. doi:10.1002/chem.201501101

Hao, L. Y., Hu, X. H., Wang, H. Q., Yang, X. L., Zhang, G. J., Wang, G. Y., et al. (2011). Int. J. Polym. Sci. ID, 814163.

Hoteling, A. J., Nichols, W. F., Harmon, P. S., Conlon, S. M., Nuñez, I. M., Hoff, J. W., et al. (2018). Characterization and Quantitation of PVP Content in a Silicone Hydrogel Contact Lens Produced by Dual-phase Polymerization Processing. J. Biomed. Mater. Res. 106, 1064-1072. doi:10.1002/jbm.b.33904

$\mathrm{Hu}, \mathrm{X}$., and Gong, X. (2016). A New Route to Fabricate Biocompatible Hydrogels with Controlled Drug Delivery Behavior. J. Colloid Interf. Sci. 470, 62-70. doi:10.1016/j.jcis.2016.02.037

Kim, D.-Y., Lee, S.-A., Park, M., Choi, Y.-J., Kang, S.-W., and Jeong, K.-U. (2015). Multi-responsible Chameleon Molecule with Chiral Naphthyl and Azobenzene Moieties. Soft matter 11, 2924-2933. doi:10.1039/c5sm00073d

Li, Z., Wang, P., Liu, B., Wang, Y., Zhang, J., Yan, Y., et al. (2014). Unusual, PhotoInduced Self-Assembly of Azobenzene-Containing Amphiphiles. Soft matter 10, 8758-8764. doi:10.1039/c4sm01395f

Liu, G.-F., Ji, W., Wang, W.-L., and Feng, C.-L. (2015). Multiresponsive Hydrogel Coassembled from Phenylalanine and Azobenzene Derivatives as 3D Scaffolds for Photoguiding Cell Adhesion and Release. ACS Appl. Mater. Inter. 7, 301-307. doi:10.1021/am506202s

Lorente-Velazquez, A., Garcia-Montero, M., Gomez-Sanz, F. J., Rico Del Viejo, L., Hernandez-Verdejo, J. L., and Madrid-Costa, D. (2019). Int. J. Ophthalmol. 12, 640-646.

\section{AUTHOR CONTRIBUTIONS}

XL prepared the hydrogel HZ investigated the properties of materials $\mathrm{YQ}$ evaluated the drug release properties $\mathrm{XH}$ give the whole idea.

\section{FUNDING}

This study was financially supported by Natural Science Foundation of China (21702082), Natural Science Foundation of Jiangsu Province (BK20171113).

Lorenzo, R. A., Carro, A. M., Concheiro, A., and Alvarez-Lorenzo, C. (2015). Stimuli-responsive Materials in Analytical Separation. Anal. Bioanal. Chem. 407, 4927-4948. doi:10.1007/s00216-015-8679-1

Pang, J., Gao, Z., Zhang, L., Wang, H., and Hu, X. (2018). Front. Chem. 6 (ID), 217. doi:10.3389/fchem.2018.00217

Pang, J., Kong, J. Y., Xu, J. L., Mao, X. C., and Hu, X. H. (2020). Front. Mater. 7. ID, 120. doi: $10.3389 /$ fmats. 2020.00120

Pang, J., Mao, X., Xu, J., Zhao, X., Kong, J., and Hu, X. (2020). Design, Synthesis, and Investigation of a Visible Light-Driven Photo-Switching Macromolecule. RSC Adv. 10, 35164-35173. doi:10.1039/d0ra06627c

Pang, J., Tian, Z., and Ma, J. (2014). Theoretical Design of Visible Light Driven Azobenzene-Based Photo-Switching Molecules. Chem. Phys. Lett. 613, 110-114. doi:10.1016/j.cplett.2014.07.048

Samsom, M., Korogiannaki, M., Subbaraman, L. N., Sheardown, H., and Schmidt, T. A. (2018). Hyaluronan Incorporation into Model Contact Lens Hydrogels as a Built-In Lubricant: Effect of Hydrogel Composition and Proteoglycan 4 as a Lubricant in Solution. J. Biomed. Mater. Res. 106, 1818-1826. doi:10.1002/jbm.b.33989

Tong, W., Karthik, N., Li, J., Wang, N., and Xiong, D. (2019). Superhydrophobic Surface with Stepwise Multilayered Micro- and Nanostructure and an Investigation of its Corrosion Resistance. Langmuir 35, 15078-15085. doi:10.1021/acs.langmuir.9b02910

Wang, D., Wagner, M., Butt, H.-J., and Wu, S. (2015). Supramolecular Hydrogels Constructed by Red-Light-Responsive Host-Guest Interactions for PhotoControlled Protein Release in Deep Tissue. Soft matter 11, 7656-7662. doi: $10.1039 / \mathrm{c} 5 \mathrm{sm} 01888 \mathrm{a}$

Wei, D. W., Wei, H., Gauthier, A. C., Song, J., Jin, Y., and Xiao, H. (2020) Superhydrophobic Modification of Cellulose and Cotton Textiles: Methodologies and Applications. J. Bioresources Bioproducts 5, 1-15. doi:10.1016/j.jobab.2020.03.001

Wei, S., Yin, R., Tang, T., Wu, Y., Liu, Y., Wang, P., et al. (2019). Gas-Permeable, Irritation-free, Transparent Hydrogel Contact Lens Devices with Metal-Coated Nanofiber Mesh for Eye Interfacing. ACS nano 13, 7920-7929. doi:10.1021/ acsnano.9b02305

Xiao, Y., Tang, C., Chen, Y., and Lang, M. (2019). Dual Stimuli-responsive Polypeptide Prepared by Thiol-ene Click Reaction of Poly( L -cysteine) and N, N -dimethylaminoethyl Acrylate. Biopolymers 110, e23318. doi:10.1002/bip.23318

Conflict of Interest: The authors declare that the research was conducted in the absence of any commercial or financial relationships that could be construed as a potential conflict of interest.

Publisher's Note: All claims expressed in this article are solely those of the authors and do not necessarily represent those of their affiliated organizations, or those of the publisher, the editors and the reviewers. Any product that may be evaluated in this article, or claim that may be made by its manufacturer, is not guaranteed or endorsed by the publisher.

Copyright (c) 2021 Lin, Zhang, Qin and Hu. This is an open-access article distributed under the terms of the Creative Commons Attribution License (CC BY). The use, distribution or reproduction in other forums is permitted, provided the original author(s) and the copyright owner(s) are credited and that the original publication in this journal is cited, in accordance with accepted academic practice. No use, distribution or reproduction is permitted which does not comply with these terms. 